\title{
Singularities in the Hamiltonian at electronic degeneracies
}

\author{
A.J.C. Varandas *, Z.R. Xu \\ Departamento de Química, Universidade de Coimbra, P-3049 Coimbra Codex, Portugal \\ Received 29 March 2000; in final form 13 May 2000
}

\begin{abstract}
We discuss the singularities which arise in the Hamiltonian operator at a crossing seam involving two potential energy surfaces of the same global symmetry. The Mead-Truhlar and our own equations are discussed and found to differ from each other, although leading to identical phases up to a constant factor and sign in the vicinity of the crossing seam. Also established are the relations which link the vector and scalar gauge potentials with the mixing angle. (c) 2000 Elsevier Science B.V. All rights reserved.
\end{abstract}

\section{Introduction}

About 40 years ago, Herzberg and LonguetHiggins [1] showed that a real-valued electronically adiabatic wave function changes sign when the nuclear coordinates traverse a closed path encircling a conical intersection. This implies that the standard Born-Oppenheimer (BO) treatment [2] breaks down whenever such a conical intersection $[1,3-8]$ is present. Twenty years ago, Mead and Truhlar $[9,10]$ generalized the BO method by introducing [9-11] a vector potential into the nuclear Schrödinger equation. A few years later, Berry [12] examined the adiabatic evolution of a quantum system and discovered in a wider context what is usually referred to as the geometric phase (GP) effect (also known as the Berry's phase effect and molecular Aharonov-Bohm [13] effect; for reviews, see Refs. [14,15]). Such an effect may have important implications in many fields, including

\footnotetext{
* Corresponding author. Fax: +351-39-27703.

E-mail address: varandas@qtvsl.uc.pt (A.J.C. Varandas).
}

molecular spectra [16-20] and scattering [21-27], and hence became a topic of considerable research activity $[14,15,28]$.

Although the GP effect has been mostly studied in homonuclear triatomic systems $\left(\mathrm{X}_{3}\right)$, it has been shown [29] by ab initio methods to be also generally present in systems without permutational symmetry such as LiNaK. For $\mathrm{X}_{3}$ molecules, the GP effect can be studied by multiplying the real double-valued electronic wave function by a complex phase-factor which shows a compensating change of sign on encircling the conical intersection such as to make the total electronuclear wave function continuous [24,30-32]. This approach leads to the above mentioned vector potential $[9,10]$. Instead, Billing and Markovic [33] adopted hyperspherical coordinates to include the GP effect in the nuclear wave function of $\mathrm{X}_{3}$ molecules, which have a $\mathrm{D}_{3 \mathrm{~h}}$ conical intersection seam. A similar method has been utilized by the authors to study the vibrational spectra and transition state resonances of $\mathrm{H}_{3}$ [16] and $\mathrm{Li}_{3}[18,19]$. Such an approach has most recently been extended to isotopomers of $\mathrm{X}_{3}$ systems [34]. 
A few other developments in the theory should be mentioned at this point. In 1997, Baer [35] suggested a connection between the first derivative coupling term and the geometric phase (for a critical comment to his work, see Ref. [36]), while we studied the behavior of single-surface nuclear wave functions in the vicinity of the conical intersection for a $\mathrm{X}_{3}$ system [37]. In turn, the authors and Baer [38] used the line-integral technique [35,39-49] to study the GP effect in coupled two-state systems. We have then found that the adiabatic-diabatic-transformation angle $\alpha(\mathbf{R})$ is identical (up to a constant) to the mixing angle of the orthogonal transformation which diagonalizes the diabatic potential matrix [50-55]. Most recently, we derived [56] a novel generalized BornOppenheimer equation for a two coupled-state problem in the vicinity of the crossing seam, and found that the geometric phase angle is identical (up to a constant) to the mixing angle.

The paper is arranged as follows. In Section 2, which comprises three subsections, we discuss the singularities which occur in the Hamiltonian at a conical intersection by considering different approaches to the treatment of the GP effect in coupled two-state models. The concluding remarks are in Section 3.

\section{Theory}

\subsection{Singularities at crossing seams}

As pointed out in the Introduction, the GP effect can be studied by multiplying the real doublevalued electronic wave function by a complex phase-factor which changes sign on encircling the conical intersection such that the resulting total electronuclear wave function becomes single-valued. Following Mead and Truhlar [5,9,10,21,32] one writes

$\tilde{\psi}=\exp [\mathrm{i} \beta(\mathbf{R})] \psi$,

where $\psi$ is the real adiabatic electronic wave function, $\beta(\mathbf{R})$ is the geometric phase, and $\mathbf{R}$ denotes the nuclear internal degrees of freedom; for brevity, we will suppress heretofore an obvious dependence of the electronic wave function on the electrons and nuclear coordinates (in this case it is only a parametric dependence), and that of the nuclear wave function on the nuclear coordinates. Note that $\beta(\mathbf{R})$ must change by $\pi$ for any nuclear motion which encircles the conical intersection (i.e., a pseudo-rotation). In order to meet this boundary condition, it is commonly used $[4,9,10$, $16,18,19,23,25,32,57-61]$ for $\mathrm{X}_{3}$ systems the form

$\beta(\mathbf{R})=\frac{\varphi}{2}$,

where $\varphi$ is the pseudo-rotation angle. Note also that Eq. (1) represents a gauge transformation on the electronic wave function, and hence the geometric phase $\beta(\mathbf{R})$ may differ by a multiple of a half odd-integer.

Using the complex electronic wave function described above, one obtains the generalized Born-Oppenheimer equation $[9,10,59,60,62]$

$\left\{\frac{\hbar^{2}}{2 \mu}\left[-\nabla^{2}+\mathbf{A}^{2}+\mathrm{i} \nabla \mathbf{A}+\mathrm{i} \mathbf{A} \cdot \nabla\right]+\Phi+V\right\} \bar{\chi}=E \bar{\chi}$,

where $\mu$ is a characteristic reduced mass of the system [63], $\bar{\chi}$ is the real-valued nuclear wave function, and $\mathbf{A}$ and $\Phi$ are the magnetic vector and electric scalar gauge potentials defined by $[9,62]$

$\mathbf{A}(\mathbf{R})=\mathrm{i}\langle\tilde{\psi} \mid \nabla \tilde{\psi}\rangle$

and $[21,62]$

$\Phi(\mathbf{R})=-\frac{\hbar^{2}}{2 \mu} \sum_{m \neq 1}\left\langle\tilde{\psi}_{1} \mid \nabla \tilde{\psi}_{m}\right\rangle\left\langle\tilde{\psi}_{m} \mid \nabla \tilde{\psi}_{1}\right\rangle$.

In the following, we derive more compact formulas of $\mathbf{A}(\mathbf{R})$ and $\Phi(\mathbf{R})$ for a $2 \mathrm{D}$ Hilbert space model, i.e., for two intersecting adiabatic potential energy surfaces. First, we replace Eq. (1) into Eq. (4), and note that $\langle\psi \mid \nabla \psi\rangle=0$. The result is

$\mathbf{A}(\mathbf{R})=-\nabla \beta(\mathbf{R})$

which provides an explicit relation between the magnetic vector potential and the geometric phase. We then express the two real-valued orthonormalized electronic states as [56] 
$\psi_{1}=\left[\begin{array}{c}\cos \gamma(\mathbf{R}) \\ -\sin \gamma(\mathbf{R})\end{array}\right], \quad \psi_{2}=\left[\begin{array}{c}\sin \gamma(\mathbf{R}) \\ \cos \gamma(\mathbf{R})\end{array}\right]$,

where $\gamma(\mathbf{R})$ is the mixing angle [38,51-56] which diagonalizes the potential matrix. One gets [56]

$\nabla \psi_{1}=-\psi_{2} \nabla \gamma(\mathbf{R})$,

$\nabla \psi_{2}=\psi_{1} \nabla \gamma(\mathbf{R})$

and hence

$\left\langle\psi_{1} \mid \nabla \psi_{2}\right\rangle=\nabla \gamma(\mathbf{R})$,

$\left\langle\psi_{2} \mid \nabla \psi_{1}\right\rangle=-\nabla \gamma(\mathbf{R})$.

Thus, using Eqs. (1), (10) and (11), Eq. (5) assumes the form

$\Phi(\mathbf{R})=\frac{\hbar^{2}}{2 \mu}(\nabla \gamma)^{2}$

which provides an explicit connection between the scalar electric potential and the mixing angle. Note that formal expressions for the scalar electric potential have been derived before (see, e.g., Ref. [64]), although the connection to the mixing angle is to our knowledge presented here for the first time. Because the mass $\mu$ is positive and $\gamma$ is real, the electric scalar potential becomes repulsive as first pointed out by Berry and Lim [62].

We now examine the behavior of the vector and scalar gauge potentials at the conical intersection. As shown by Kendrick and Pack [21] using hyperspherical coordinates $[65,66]$, the vector components of $\mathbf{A}(\rho, \theta, \phi)$ assume in the vicinity of a conical intersection the form

$A_{\rho}=0$,

$A_{\theta} \approx \frac{\sin \theta_{\mathrm{s}} \delta \phi}{2\left(\delta \theta^{2}+\sin ^{2} \theta_{\mathrm{s}} \delta \phi^{2}\right)}$,

$A_{\phi} \approx-\frac{\sin \theta_{\mathrm{s}} \delta \theta}{2\left(\delta \theta^{2}+\sin ^{2} \theta_{\mathrm{s}} \delta \phi^{2}\right)}$,

where $\delta \theta$ and $\delta \phi$ measure the deviations of $\theta$ and $\phi$ from the conical intersection, which occurs for $\theta=\theta_{\mathrm{s}}$ and $\phi=\phi_{\mathrm{s}}$. Thus, $\lim _{\delta \theta \rightarrow 0} A_{\theta}=\frac{1}{2 \sin \theta_{\mathrm{s}} \delta \phi}$,

$\lim _{\delta \phi \rightarrow 0} A_{\phi}=-\frac{\sin \theta_{\mathrm{s}}}{2 \delta \theta}$

which shows that $A_{\theta}$ and $A_{\phi}$ components have a singular behavior for $\delta \theta \rightarrow 0$ and $\delta \phi \rightarrow 0$. In fact, they show different types of singular behavior depending on the symmetry of the conical intersection locus, as we will discuss now in more detail. For a $D_{3 \mathrm{~h}}$ type conical intersection where $\theta_{\mathrm{s}}=0$ [38], $A_{\theta}$ has a singularity of order 1 both in $\theta$ and $\phi$, while $A_{\phi}$ may not be singular there. However, for a $\mathrm{C}_{2 \mathrm{v}}$ crossing seam where $\theta_{\mathrm{s}} \neq 0$ [38], $A_{\theta}$ has a singularity of order 1 in $\phi$ while $A_{\phi}$ has a singularity of order 1 in $\theta$ at $\theta=\theta_{\mathrm{s}}$.

To investigate the singular behavior of the scalar gauge potential, consider without loss of generality the Hamiltonian operator $\hat{H}_{\mathrm{e}}$ of an electronic system with a $\mathrm{D}_{3 \mathrm{~h}}$ degeneracy. Let $\psi_{1}$ and $\psi_{2}$ be the two electronic states which belong to the degenerate electronic states of $E$ symmetry. $\hat{H}_{\mathrm{e}}$ can then be expanded using the set of coordinates $\left(Q_{A_{1}}, Q_{A_{2}}\right.$, $\left.Q_{E_{x}}, Q_{E_{y}}\right)$ which belong to the irreducible representations $A_{1}, A_{2}, E_{x}, E_{y}$ of the $\mathrm{D}_{3 \mathrm{~h}}$ point group [20]

$\hat{H}_{\mathrm{e}}=\left[\begin{array}{llll}\hat{h}_{A_{1}} & \hat{h}_{A_{2}} & \hat{h}_{E_{x}} & \hat{h}_{E_{y}}\end{array}\right]\left[\begin{array}{l}Q_{A_{1}} \\ Q_{A_{2}} \\ Q_{E_{x}} \\ Q_{E_{y}}\end{array}\right]$,

where $\hat{h}_{X}\left(X=A_{1}, A_{2}, E_{x}, E_{y}\right)$ are expansion coefficients representing electronic operators which are independent of the nuclear coordinates. We may now define complex coordinates and electronic states according to Ref. [5].

$Q_{+}=Q_{E_{x}}+\mathrm{i} Q_{E_{y}}=\rho \exp (\mathrm{i} \phi)$,

$Q_{-}=Q_{E_{x}}-\mathrm{i} Q_{E_{y}}=\rho \exp (-\mathrm{i} \phi)$

and

$\psi_{+}=\psi_{1}+\mathrm{i} \psi_{2}$

$\psi_{-}=\psi_{1}-\mathrm{i} \psi_{2}$

The $E$ representation of the electronic Hamiltonian can then be expressed as 
$\mathbf{W}=\left\langle E\left|\hat{H}_{\mathrm{e}}\right| E\right\rangle=\left[\begin{array}{llll}\left\langle E\left|\hat{h}_{A_{1}}\right| E\right\rangle & \left\langle E\left|\hat{h}_{A_{2}}\right| E\right\rangle & \left\langle E\left|\hat{h}_{E_{x}}\right| E\right\rangle & \left\langle E\left|\hat{h}_{E_{y}}\right| E\right\rangle\end{array}\right]\left[\begin{array}{c}Q_{A_{1}} \\ Q_{A_{2}} \\ Q_{E_{x}} \\ Q_{E_{y}}\end{array}\right]$

with $[20,57]$

$\left\langle E\left|\hat{h}_{A_{1}}\right| E\right\rangle=\omega_{A_{1}}\left[\begin{array}{ll}1 & 0 \\ 0 & 1\end{array}\right]=\omega_{A_{1}} \mathbf{I}$,

$\left\langle E\left|\hat{h}_{A_{2}}\right| E\right\rangle=\omega_{A_{2}}\left[\begin{array}{cc}0 & -1 \\ 1 & 0\end{array}\right]=-\mathrm{i} \omega_{A_{2}} \boldsymbol{\sigma}_{2}$

$\left\langle E\left|\hat{h}_{E_{x}}\right| E\right\rangle=\omega_{E_{x}}\left[\begin{array}{cc}-1 & 0 \\ 0 & 1\end{array}\right]=-\omega_{E_{x}} \boldsymbol{\sigma}_{3}$,

$\left\langle E\left|\hat{h}_{E_{y}}\right| E\right\rangle=\omega_{E_{y}}\left[\begin{array}{ll}0 & 1 \\ 1 & 0\end{array}\right]=\omega_{E_{y}} \boldsymbol{\sigma}_{1}$,

where $\omega_{X}$ are constants, $\boldsymbol{\sigma}=\left(\boldsymbol{\sigma}_{1}, \boldsymbol{\sigma}_{2}, \boldsymbol{\sigma}_{3}\right)$ are the Pauli spin matrices, and $\mathbf{I}$ is the $2 \times 2$ identity matrix. Because the Hamiltonian $\hat{H}_{\mathrm{e}}$ is required to be Hermitian, we must now remove the term involving $\boldsymbol{\sigma}_{2}$. Defining next a set of scaled coordinates $\mathbf{X}=\left(X_{1}, X_{2}, X_{3}\right)$ as

$X_{1}=\omega_{A_{1}} Q_{A_{1}}$,

$X_{2}=-\omega_{E_{x}} Q_{E_{x}}$,

$X_{3}=\omega_{E_{y}} Q_{E_{y}}$,

one obtains

$\mathbf{W}=\left(\begin{array}{lll}\mathbf{I} & \boldsymbol{\sigma}_{3} & \boldsymbol{\sigma}_{1}\end{array}\right)\left(\begin{array}{l}X_{1} \\ X_{2} \\ X_{3}\end{array}\right)$,

which can also be written in matrix form as

$\mathbf{W}=\overline{\boldsymbol{\sigma}} \cdot \mathbf{X}$

where $\overline{\boldsymbol{\sigma}}=\left(\mathbf{I} \boldsymbol{\sigma}_{3} \boldsymbol{\sigma}_{1}\right)$.

Diagonalization of $\mathbf{W}$ then leads to the electronically adiabatic potential energies

$V_{ \pm}=X_{1} \pm \sqrt{X_{2}^{2}+X_{3}^{2}}$.

Noting now that
$\omega_{E_{x}}=\left\langle\psi_{+}\left|\hat{h}_{E_{x}}\right| \psi_{-}\right\rangle$,

$\omega_{E_{y}}=\left\langle\psi_{-}\left|\hat{h}_{E_{y}}\right| \psi_{+}\right\rangle$,

one obtains $\omega_{E}=\omega_{E_{x}}=\omega_{E_{y}}$. By replacing this result into Eqs. (29) and (30), one gets from Eq. (33) the familiar form $[57,67]$

$V_{ \pm}=X_{1} \pm \omega_{E} \rho$,

where we have also used Eqs. (19) and (20). We now follow Berry and Lim [62] using our coordinates to write the quantum metric tensor [68] as

$\mathbf{g}(\mathbf{X})=\frac{1}{4 \omega_{E}^{2} \rho^{2}}\left[\begin{array}{lll}0 & 0 & 0 \\ 0 & 1 & 0 \\ 0 & 0 & 1\end{array}\right]$.

Since the scalar gauge potential can take the form [62]

$\Phi(\mathbf{X})=\frac{\hbar^{2}}{2} q^{i j} g_{i j}(\mathbf{X})$,

where $q^{i j}$ is the $i j$ element of the inverse mass tensor $\mathbf{q}^{-1}$ (note that a summation over all $i$ and $j$ is implied in Eq. (38)), one gets

$\Phi(\mathbf{X})=\frac{\hbar^{2}}{4 \mu \omega_{E}^{2} \rho^{2}}$.

Clearly, the electric scalar gauge potential has a singularity of order 2 in $\rho$ at the conical intersection.

\subsection{Single-surface generalized Born-Oppenheimer equation}

An equation distinct from Eq. (3) has been derived by the authors [56] for the two coupled states problem by assuming that the nuclear dynamics in the vicinity of the crossing seam plays the dominant role. It differs from one derived earlier by Baer and coworkers [64,69] for the lower adiabatic sheet in that the mixing angle appears now ex- 
plicitly in it. Moreover, our equation has been derived under the assumption that the dynamics is controlled by the vicinity of the conical intersection, where all approximations involved in its derivation are justified [56]. Thus, it is valid to describe also the nuclear dynamics in the upper adiabatic potential energy surface provided that one uses the appropriate adiabatic potential function. Such a single surface equation bears the form

$$
\left\{\frac{\hbar^{2}}{2 \mu}\left[-\nabla^{2}+(\nabla \gamma)^{2}+\mathrm{i} \nabla^{2} \gamma+\mathrm{i} 2 \nabla \gamma \cdot \nabla\right]+V\right\} \tilde{\chi}=E \tilde{\chi},
$$

where $\gamma(\mathbf{R})$ is the mixing angle referred to in Section 2.1, and the complex nuclear wave function is defined by

$\tilde{\chi}=\frac{1}{\sqrt{2}}\left(\chi_{1}+\mathrm{i} \chi_{2}\right)$

with $\chi_{n}(n=1,2)$ are the real-valued nuclear wave functions associated to the two crossing electronically adiabatic potential energy surfaces. Such a complex nuclear wave function may also be written as

$\tilde{\chi}=\exp [\mathrm{i} A(\mathbf{R})] \chi$,

where $A(\mathbf{R})$ is a geometric phase (this should not be confused with the magnetic vector potential) in principle distinct from $\beta(\mathbf{R})$. By associating the phase to the real-valued electronic wave function, we may also write [56]

$\tilde{\psi}=\exp [\mathrm{i} A(\mathbf{R})] \psi$.

We should recall at this point that the geometric phase $A(\mathbf{R})$ has been shown to be identical (up to a constant) to the mixing angle $\gamma(\mathbf{R})$, and $\alpha(\mathbf{R})$ [56].

Clearly, Eq. (40) here referred to for brevity as the Varandas-Xu (VX) equation is different from the Mead-Truhlar (MT) one in Eq. (3). However, they both describe properly the geometric phase effect, although being based on phases $A(\mathbf{R})$ and $\beta(\mathbf{R})$ which are in principle distinct from each other. In fact, by comparing Eqs. (1) and (43) and having in mind that the sign of the phase is irrelevant, one may write
$A(\mathbf{R})=-\beta(\mathbf{R})+C$,

and hence

$\nabla A(\mathbf{R})=-\nabla \beta(\mathbf{R})$.

Note that $A(\mathbf{R})$ must be equal (up to a constant) to the geometric phase, since both Eqs. (40) and (4) are valid in the vicinity of the crossing seam. In fact, this observation has been used to select the sign in Eq. (44) (note that the constant $C$ in Eq. (44) is arbitrary, and may be chosen to vanish without loss of generality). Thus, $A(\mathbf{R})$ and $\beta(\mathbf{R})$ are identical up to a sign, which is irrelevant since it implies only a pseudo-rotation in the opposite direction. Moreover, from Eqs. (6) and (45) and noting that $\nabla A(\mathbf{R})=\nabla \gamma(\mathbf{R})$ [56], the vector gauge potential assumes the form

$\mathbf{A}(\mathbf{R})=\nabla \gamma(\mathbf{R})$.

The singularities at the crossing seam of the VX equation come therefore from the terms $\nabla \gamma$ and $(\nabla \gamma)^{2}$. In fact, by comparing Eqs. (12) and (39), one obtains in polar coordinates

$(\nabla \gamma)^{2}=\frac{1}{2 \omega_{E}^{2} \rho^{2}}$.

This shows that $\nabla \gamma$ has a singularity of order 1 in $\rho$, and $(\nabla \gamma)^{2}$ a singularity of order 2 in $\rho$. Such a conclusion should hold also for other types of coordinates, i.e., $\nabla \gamma$ has a singularity of order 1 , and $(\nabla \gamma)^{2}$ a singularity of order 2 , along a given coordinate.

\subsection{Effects of singularities at the conical intersec- tion}

The radial component $A_{\rho}$ of the vector gauge potential $\mathbf{A}(\mathbf{R})$ is zero, and hence it does not affect the nuclear wave function. This result is corroborated from calculations of the vibrational spectrum for the ground and the first excited states of $\mathrm{Li}_{3}[18,19]$. In fact, we have then found the fundamental vibrational frequency of the symmetric stretching mode to be insensitive to inclusion of the geometric phase effect. Conversely, the calculated fundamental vibrational frequency of the degenerate mode has shown a remarkable variation upon inclusion of the GP effect. This demonstrates 
that the angular components of the vector gauge potential $\mathbf{A}(\mathbf{R})$ play an important role.

The scalar gauge potential is proportional to $\hbar^{2}$ and gives negligible contributions to one-dimensional subsequences of vibronic levels. However, it cannot be ignored near a degeneracy. In fact, as pointed out by Berry and Lim [62], its effect will be to improve the adiabatic approximation by repelling the slow system from degeneracies and reducing the probability of nonadiabatic transitions between electronic states.

Finally, the occurrence of singularities at the conical intersection prevent the nuclear wave functions from penetrating in the vicinity of the conical intersection. In fact, we have found [37] that the nuclear wave function assumes there the form

$$
\begin{aligned}
\chi(\rho, \theta, \phi) \rightarrow & \exp (-\sqrt{\epsilon} \rho) \rho^{v} F(\rho) P_{l}^{|m|}(\cos \theta) \\
& \times \exp (\mathrm{i} m \phi),
\end{aligned}
$$

where $v=1,2, \ldots$, and

$\lim _{\rho \rightarrow 0} F(\rho)={ }_{1} F_{1}(2(v+1) \sqrt{\epsilon}, 2(v+1), \rho)$,

where ${ }_{1} F_{1}(\cdots)$ is the Kummer's function or confluent hypergeometric function. Thus, they vanish at the conical intersection as $\rho^{v}$, a result which has been numerically verified for $\mathrm{Li}_{3}$. Because the singularities in the Hamiltonian at the conical intersection have at most order 2 in the chosen coordinates, the Hamiltonian matrix elements will therefore vanish there with order $2 v-1$, i.e., at least order 1 if we take into account that the nuclear wave function has at least order 1 in Eq. (48). In other words, the Hamiltonian matrix elements vanish at the conical intersection.

\section{Concluding remarks}

In the present work, we have examined the singularities which arise at the crossing seam in the single-surface treatment of the quantum dynamics. The MT and VX equations (Eqs. (3) and (40), respectively) have been shown to differ from each other, although leading to the same phase angles. The relations connecting the magnetic vector and electric scalar gauge potentials with the mixing angle have also been determined. Finally, the behavior of the title singularities and their implications in the nuclear dynamics have been analyzed.

\section{Acknowledgements}

This work has been supported by the Fundação para a Ciência e Tecnologia, Portugal, under programme PRAXIS XXI.

\section{References}

[1] G. Herzberg, H.C. Longuet-Higgins, Faraday Discuss. Chem. Soc. 35 (1963) 77.

[2] M. Born, J.R. Oppenheimer, Ann. Phys. 84 (1927) 457.

[3] H.A. Jahn, E. Teller, Proc. R. Soc. 161A (1937) 220.

[4] H.C. Longuet-Higgins, U. Öpik, M.H.L. Pryce, R.A. Sack, Proc. R. Soc. 244A (1958) 1.

[5] H.C. Longuet-Higgins, Adv. Spectrosc. 2 (1961) 429.

[6] M.S. Child, H.C. Longuet-Higgins, Phyl. Trans. R. Soc. London A 254 (1961) 259.

[7] H.C. Longuet-Higgins, Proc. R. Soc. Lond A 344 (1975) 147.

[8] H. Köppel, W. Domcke, L.S. Cederbaum, Adv. Chem. Phys. 72 (1984) 59.

[9] C.A. Mead, D.G. Truhlar, J. Chem. Phys. 70 (1979) 2284.

[10] C.A. Mead, J. Chem. Phys. 72 (1980) 3839.

[11] C.A. Mead, Chem. Phys. 49 (1980) 23.

[12] M.V. Berry, Proc. R. Soc. A392 (1984) 45.

[13] Y. Aharonov, B. Bohm, Phys. Rev. 115 (1959) 485.

[14] C.A. Mead, Rev. Mod. Phys. 64 (1992) 51.

[15] D.R. Yarkony, Rev. Mod. Phys. 68 (1996) 985.

[16] A.J.C. Varandas, H.G. Yu, J. Chem. Soc. Faraday Trans. 93 (1997) 819.

[17] B.K. Kendrick, Phys. Rev. Lett. 79 (1997) 2431.

[18] A.J.C. Varandas, H.G. Yu, Z.R. Xu, Mol. Phys. 96 (1999) 1193.

[19] A.J.C. Varandas, Z.R. Xu, Int. J. Quant. Chem. 75 (1999) 89.

[20] A.J.C. Varandas, Z.R. Xu, in: Progress in Theoretical Chemistry and Physics, R. Bader, L. Montero (Eds.), Kluwer, Dordrecht, 2000.

[21] B.K. Kendrick, R.T Pack, J. Chem. Phys. 104 (1996) 7475.

[22] B. Lepetit, Z. Peng, A. Kuppermann, Chem. Phys. Lett. 166 (1990) 572.

[23] B. Lepetit, A. Kuppermann, Chem. Phys. Lett. 166 (1990) 581.

[24] Y.-S.M. Wu, B. Lepetit, A. Kuppermann, Chem. Phys. Lett. 186 (1991) 319. 
[25] A. Kuppermann, Y.-S.M. Wu, Chem. Phys. Lett. 205 (1993) 577.

[26] A. Kuppermann, Y.-S.M. Wu, Chem. Phys. Lett. 241 (1995) 229.

[27] L. Schnieder, K. Seekamp-Rahn, J. Borkowski, E. Wrede, K.H. Welge, F.J. Aoiz, L. Banares, M.J. D’Mello, V.J. Herrero, V. Saez Rabanos, R.E. Wyatt, Science 269 (1995) 207.

[28] M. Baer, J. Chem. Phys. 109 (1998) 891.

[29] A.J.C. Varandas, J. Tennyson, J.N. Murrell, Chem. Phys. Lett. 61 (1979) 431.

[30] Y.-S.M. Wu, A. Kuppermann, Chem. Phys. Lett. 201 (1993) 178.

[31] A. Kuppermann, Y.-S.M. Wu, Chem. Phys. Lett. 213 (1993) 636.

[32] T.C. Thompson, D.G. Truhlar, C.A. Mead, J. Chem. Phys. 82 (1985) 2392.

[33] G.D. Billing, N. Marković, J. Chem. Phys. 99 (1993) 2674.

[34] Z.R. Xu, A.J.C. Varandas, Int. J. Quant. Chem., in press.

[35] M. Baer, J. Chem. Phys. 107 (1997) 2694.

[36] B.K. Kendrick, C.A. Mead, D.G. Truhlar, J. Chem. Phys. 110 (1999) 7594.

[37] A.J.C. Varandas, Z.R. Xu, Chem. Phys. Lett. 316 (2000) 248.

[38] Z.R. Xu, M. Baer, A.J.C. Varandas, J. Chem. Phys. 112 (2000) 2746.

[39] M. Baer, Chem. Phys. Lett. 35 (1975) 112.

[40] M. Baer, Chem. Phys. 15 (1976) 49.

[41] M. Baer, Mol. Phys. 40 (1980) 1011.

[42] M. Baer, Theory of Chemical Reaction Dynamics, in: M. Baer (Ed.), (CRC, Boca Raton, 1985), vol. II, pp. 243-244.

[43] M. Baer, R. Englman, Mol. Phys. 75 (1992) 293.

[44] T. Pacher, L.S. Cederbaum, H. Köppel, Adv. Chem. Phys. 84 (1993) 293

[45] W. Domcke, G. Stock, Adv. Chem. Phys. 100 (1997) 1.

[46] D.R. Yarkony, J. Chem. Phys. 105 (1996) 10456.
[47] R.G. Sadygov, D.R. Yarkony, J. Chem. Phys. 109 (1998) 20.

[48] N. Matsunaga, D.R. Yarkony, J. Chem. Phys. 107 (1997) 7825.

[49] G. Chaban, M.S. Gordon, D.R. Yarkony, J. Phys. Chem. 101A (1997) 7953.

[50] A.J.C. Varandas, H.G. Yu, Chem. Phys. Lett. 259 (1996) 336.

[51] A.J.C. Varandas, J. Chem. Phys. 107 (1997) 867.

[52] A.J.C. Varandas, A.I. Voronin, P.J.S.B. Caridade, J. Chem. Phys. 108 (1998) 7623.

[53] R.K. Preston, J.C. Tully, J. Chem. Phys. 54 (1971) 4297.

[54] D. Grimbert, B. Lassier-Govers, V. Sidis, Chem. Phys. 124 (1988) 187.

[55] F. Gianturco, A. Palma, F. Schnider, Chem. Phys. 137 (1989) 177.

[56] A.J.C. Varandas, Z.R. Xu, J. Chem. Phys. 112 (2000) 2121.

[57] T.C. Thompson, C.A. Mead, J. Chem. Phys. 82 (1985) 2408.

[58] J.T. Muckermann, R.D. Gilbert, G.D. Billing, J. Chem. Phys. 88 (1988) 4779.

[59] B.K. Kendrick, R.T Pack, J. Chem. Phys. 104 (1996) 7329.

[60] B.K. Kendrick, R.T Pack, J. Chem. Phys. 104 (1996) 7502.

[61] D. Yarkony, J. Chem. Phys. 110 (1999) 701.

[62] M.V. Berry, R. Lim, J. Phys. A: Math. Gen. 23 (1990) L655.

[63] L.M. Delves, Nucl. Phys. 20 (1960) 275.

[64] M. Baer, R. Englman, Chem. Phys. Lett. 265 (1997) 105.

[65] B.R. Johnson, J. Chem. Phys. 73 (1980) 5051.

[66] B.R. Johnson, J. Chem. Phys. 79 (1983) 1916.

[67] W. Moffitt, A.D. Liehr, Phys. Rev. 106 (1957) 1195.

[68] B. Schutz, Geometrical Methods of Mathematical Physics, Cambridge University, New York, 1988.

[69] R. Baer, D.M. Charutz, R. Kosloff, M. Baer, J. Chem. Phys. 105 (1996) 9141. 